\title{
Multipliers of Modules of Continuous Vector-Valued Functions
}

\author{
Liaqat Ali Khan and Saud M. Alsulami \\ Department of Mathematics, King Abdulaziz University, P.O. Box 80203, Jeddah 21589, Saudi Arabia \\ Correspondence should be addressed to Liaqat Ali Khan; 1khan@kau.edu.sa
}

Received 8 November 2013; Accepted 11 January 2014; Published 12 May 2014

Academic Editor: M. Mursaleen

Copyright (C) 2014 L. A. Khan and S. M. Alsulami. This is an open access article distributed under the Creative Commons Attribution License, which permits unrestricted use, distribution, and reproduction in any medium, provided the original work is properly cited.

\begin{abstract}
In 1961, Wang showed that if $A$ is the commutative $C^{*}$-algebra $C_{0}(X)$ with $X$ a locally compact Hausdorff space, then $M\left(C_{0}(X)\right) \cong$ $C_{b}(X)$. Later, this type of characterization of multipliers of spaces of continuous scalar-valued functions has also been generalized to algebras and modules of continuous vector-valued functions by several authors. In this paper, we obtain further extension of these results by showing that $\operatorname{Hom}_{C_{0}(X, A)}\left(C_{0}(X, E), C_{0}(X, F)\right) \simeq C_{s, b}\left(X, \operatorname{Hom}_{A}(E, F)\right)$, where $E$ and $F$ are $p$-normed spaces which are also essential isometric left $A$-modules with $A$ being a certain commutative $F$-algebra, not necessarily locally convex. Our results unify and extend several known results in the literature.
\end{abstract}

\section{Introduction}

Characterizations of multipliers on algebras and modules of continuous functions with values in a commutative Banach or $C^{*}$-algebra $A$ have been obtained by several authors. In 1961, Wang [1] showed that if $A$ is taken as the commutative $C^{*}$-algebra $C_{0}(X)$ with $X$ being a locally compact Hausdorff space, then $M\left(C_{0}(X)\right) \cong C_{b}(X)$. This result has also been generalized to vector-valued functions by several authors (see, e.g., [2-6]). In 1985, Lai [6] showed that if $X$ is a locally compact abelian group and $A$ is a commutative Banach algebra with a bounded approximate identity, then $M\left(C_{0}(X, A)\right) \cong C_{b}\left(X, M(A)_{u}\right)$. In 1992, Candeal Haro and Lai [3] had obtained

$$
\operatorname{Hom}_{C_{0}(X, A)}\left(C_{0}(X, E), C_{0}(X, F)\right) \simeq C_{s, b}\left(X, \operatorname{Hom}_{A}(E, F)\right) \text {, }
$$

in the case when $A$ is a commutative Banach algebra and $E$ and $F$ are left Banach $A$-modules.

A natural question arises is to investigate the extent to which these characterizations can be made beyond Banach modules. We will focus mainly on the nonlocally convex case by considering $A$ a commutative complete $p$-normed algebra, $0<p \leq 1$, having a minimal approximate identity and $E$ and $F$ being $F$-spaces which are also left $A$-modules.

We mention that the arguments of earlier authors relied heavily on the fact that, in the case of $A$, a Banach algebra,
$C_{0}(X, A)$ is isometrically isomorphic to the completed tensor product $C_{0}(X) \otimes_{\lambda} A$ with respect to the smallest cross norm $\lambda$ (see [2-5]). We will avoid the use of this technique as it need not work in our case. In fact, when $A$ is not locally convex, $\otimes_{\lambda}$ is no longer appropriate; even for $A$ a complete $p$-normed space, many complications arise (see [7, Section 10.4]; [8, p. 100]).

\section{Preliminaries}

In this section, we include some basic definitions and study various classes of topological algebras considered in this paper.

Definition 1 (see $[9,10])$. Let $E$ be a vector space over the field $\mathbb{K} \in\{\mathbb{R}, \mathbb{C}\}$.

(a) A function $q: E \rightarrow \mathbb{R}$ is called an F-seminorm on $E$ if it satisfies the following:

$\left(\mathrm{F}_{1}\right) q(u) \geq 0$ for all $u \in E$

$\left(\mathrm{F}_{2}\right) q(u)=0$ if $u=0$;

$\left(\mathrm{F}_{3}\right) q(\alpha u) \leq q(u)$ for all $u \in E$ and $\alpha \in \mathbb{K}$ with $|\alpha| \leq 1$

$\left(\mathrm{F}_{4}\right) q(u+v) \leq q(u)+q(v)$ for all $u, v \in E$;

$\left(\mathrm{F}_{5}\right)$ if $\alpha_{n} \rightarrow 0$ in $\mathbb{K}$, then $q\left(\alpha_{n} u\right) \rightarrow 0$ for all $u \in E$. 
(b) An $F$-seminorm $q$ on $E$ is called an $F$-norm if, for any $u \in E, q(u)=0$ implies $u=0$.

(c) An $F$-seminorm (or $F$-norm) $q$ on $E$ is called a $p$ seminorm (resp., $p$-norm), $0<p \leq 1$, if it also satisfies

$q(\alpha u)=|\alpha|^{p} q(u) \quad \forall u \in E, \alpha \in \mathbb{K}$. (p-homogeneous).

(d) If $q$ is an $F$-norm (resp., a $p$-norm) on a vector space $E$, then the pair $(E, q)$ is called an F-normed (resp., a p-normed) space.

(e) An $F$-norm (or a $p$-norm) $q$ on an algebra $A$ is called submultiplicative if

$$
q(a b) \leq q(a) q(b) \quad \forall a, b \in A .
$$

An algebra $A$ with a submultiplicative $F$-norm (resp., $p$ norm) $q$ is called an $F$-normed (resp., p-normed) algebra.

Definition 2. (1) A net $\left\{e_{\lambda}: \lambda \in I\right\}$ in a topological algebra $A$ is called an approximate identity if

$$
\lim _{\lambda} e_{\lambda} a=\lim _{\lambda} a e_{\lambda}=a \quad \forall a \in A .
$$

(2) An approximate identity $\left\{e_{\lambda}: \lambda \in I\right\}$ in an $F$-normed algebra $(A, q)$ is said to be minimal if $q\left(e_{\lambda}\right) \leq 1$ for all $\lambda \in I$.

If $E$ and $F$ are topological vector spaces over the field $\mathbb{K} \in$ $\{\mathbb{R}$ or $\mathbb{C}\}$, then the set of all continuous linear mappings $T$ : $E \rightarrow F$ is denoted by $C L(E, F)$. Clearly, $C L(E, F)$ is a vector space over $\mathbb{K}$ with the usual pointwise operations. Further, if $F=E, C L(E)=C L(E, E)$ is an algebra under composition (i.e., $(S T)(u)=S(T(u)), u \in E)$ and has the identity $I: E \rightarrow$ $E$ given by $I(u)=u(u \in E)$.

Definition 3. Let $\left(E, q_{E}\right)$ and $\left(F, q_{F}\right)$ be $p$-normed spaces. For any linear map $T: E \rightarrow F$, define

$$
\|T\|_{q_{E}, q_{F}}=\sup \left\{q_{F}(T u): u \in E, q_{E}(u) \leq 1\right\} .
$$

Then, by ([10, p. 101-102]), $T \in C L(E, F)$ if and only if $\|T\|_{q_{E}, q_{F}}<\infty$. Further, $\|\cdot\|_{q_{E}, q_{F}}$ is an $F$-norm on $C L(E, F)$ and, for any $T \in C L(E, F)$,

$$
q_{F}(T u) \leq\|T\|_{q_{E}, q_{F}} \cdot q_{E}(u) \quad \forall u \in E .
$$

In particular, if $T \in C L(E)=C L(E, E)$, we denote

$$
\|T\|_{q_{E}}:=\sup \left\{q_{E}(T(u)): u \in E, q_{E}(u) \leq 1\right\} .
$$

In this case, for any $S, T \in C L(E),\|S T\|_{q_{E}} \leq\|S\|_{q_{E}}\|T\|_{q_{E}}$; hence $\left(C L(E),\|\cdot\|_{q_{E}}\right)$ is a $p$-normed algebra.

Definition 4. Let $E$ and $F$ be topological vector spaces. The uniform operator topology $\sigma$ (resp., the strong operator topology $s)$ on $C L(E, F)$ is defined as the linear topology which has a base of neighborhoods of 0 consisting of all the sets of the form

$$
N(D, W)=\{T \in C L(A): T(D) \subseteq W\},
$$

where $D$ is a bounded (resp., finite) subset of $E$ and $W$ is a neighborhood of 0 in $F$. Clearly, $s \leq \sigma$. In particular, if $\left(A, q_{A}\right)$ is a $p$-normed algebra, then the $\sigma$-topology on $C L(A)$ is the one given by the $p$-norm $\|\cdot\|_{A_{p}}$. In this setting, the strong operator topology $s$ on $C L(A)$ is given by the family of $\left\{P_{a}\right.$ : $a \in A\}$ of $F$-seminorms, where

$$
P_{a}(T)=q_{A}(T(a)), \quad T \in C L(A) .
$$

Remark 5. If $\left(E, q_{E}\right)$ is a general $F$-algebra, then $\|T\|_{q_{E}}$ need not exist since the set $\left\{u \in E: q_{E}(u) \leq 1\right\}$ may not be bounded (see ([10, p. 8]; [11, 12]) for counterexamples).

Definition 6. Let $X$ be a Hausdorff topological space and $E$ a Hausdorff topological vector space over the field $\mathbb{K}(=\mathbb{R}$ or $\mathbb{C}$ ) with a base $\mathscr{W}$ of neighborhoods of 0 in $E$. A function $f$ : $X \rightarrow E$ is said to vanish at infinity if, for each neighborhood $W$ of 0 in $E$, there exists a compact set $K=K_{W} \subseteq X$ such that

$$
f(x) \in W \quad \forall x \in X \backslash K
$$

We will denote by $C_{b}(X, E)$ the vector space of all continuous bounded $E$-valued functions on $X$ and by $C_{0}(X, E)$ the subspace of $C_{b}(X, E)$ consisting of those functions which vanish at infinity. When $E=\mathbb{K}(=\mathbb{R}$ or $\mathbb{C})$, these spaces will be denoted by $C_{b}(X)$ and $C_{0}(X)$. Let $C_{b}(X) \otimes E$ denote the vector subspace of $C_{b}(X, E)$ spanned by the set of all functions of the form $\varphi \otimes u$, where $\varphi \in C_{b}(X), u \in E$, and

$$
(\varphi \otimes u)(x)=\varphi(x) u, \quad x \in X .
$$

We mention that, if $X$ is not locally compact, then $C_{0}(X, E)$ may be the trivial vector space $\{0\}$. For example, if $X=\mathbb{Q}$, the space of rationals, and $E=\mathbb{R}$, then $C_{0}(\mathbb{Q}, \mathbb{R})=\{0\}$.

Remarks 7. (i) If $E=A$ is an algebra, then $C_{b}(X, A)$ is also an algebra with respect to the pointwise multiplication defined by

$$
(f g)(x)=f(x) g(x), \quad x \in X
$$

(ii) If $E=A$ is a commutative algebra, then $C_{b}(X, A)$ is also commutative; in particular, $C_{b}(X)$ is a commutative algebra

(iii) If $E$ is only a vector space, then $C_{b}(X, E)$ is a $C_{b}(X)$-bimodule with respect to the module multiplications $(\varphi, f) \rightarrow \varphi \cdot f$ and $(f, \varphi) \rightarrow f \cdot \varphi$ defined by

$$
(\varphi \cdot f)(x)=\varphi(x) f(x)=(f \cdot \varphi)(x), \quad x \in X .
$$

(iv) If $E$ is a vector space and $A$ is algebra, then $C_{b}(X, E)$ is a left $A$-module with respect to the module multiplication $(a, f) \rightarrow a \cdot f$ as pointwise action:

$$
(a \cdot f)(x)=a f(x), \quad a \in A, f \in C_{b}(X, A), x \in X .
$$

In particular, $C_{0}(X, E)$ is a left $A$-module.

Definition 8. Let $X$ be a Hausdorff space and $E$ a Hausdorff topological vector space (TVS) over $\mathbb{K}(=\mathbb{R}$ or $\mathbb{C})$. The uniform topology $u$ on $C_{b}(X, E)$ is the linear topology which 
has a base of neighborhoods of 0 consisting of all sets of the form

$$
N(X, G)=\left\{f \in C_{b}(X, E): f(X) \subseteq W\right\},
$$

where $W$ is a neighborhood of 0 in $E$. In particular, if $E=$ $\left(E, q_{E}\right)$ is an $F$-normed space, the $u$-topology on $C_{b}(X, E)$ is given by the $F$-norm

$$
\|f\|_{q_{E}, \infty}=\sup _{x \in X} q_{E}(f(x)), \quad f \in C_{b}(X, E) .
$$

\section{Main Results}

In this section we extend some results of [2-6] from Banach modules to the more general setting of topological modules.

Definition 9 (cf. $[13,14])$. Let $\left(A, q_{A}\right)$ be a commutative $p$ normed algebra, and let $\left(E, q_{E}\right)$ be a $p$-normed space which is also an $A$-module in the usual algebraic sense. Then $E$ is called an isometric A-module if

$$
q_{F}(a u) \leq q_{A}(a) q_{F}(u) \quad \text { for any } a \in A, u \in E .
$$

If $\left(A, q_{A}\right)$ has a minimal approximate identity $\left\{e_{\lambda}: \lambda \in I\right\}$, then $E$ is called an essential $A$-module if $\lim _{\lambda} e_{\lambda} u=\lim _{\lambda} u e_{\lambda}=$ $u$ for all $u \in E$.

Definition 10. Let $\left(A, q_{A}\right)$ be a commutative $p$-normed algebra, and let $E=\left(E, q_{E}\right)$ and $F=\left(F, q_{F}\right)$ be $p$-normed spaces which are also $A$-modules. One writes

$$
\begin{aligned}
\operatorname{Hom}_{A}(E, F)=\{T \in C L(E, F): & \\
& T(a \cdot u)=a \cdot T(u) \text { for any } a \in A, u \in E\} .
\end{aligned}
$$

If $E$ is an $A$-bimodule, then defining $a * T$ by

$$
(a * T)(u)=T(u \cdot a) \quad(a \in A, u \in E),
$$

$\operatorname{Hom}_{A}(E, F)$ becomes a left $A$-module. In fact, for any $a, b \in$ $A, u \in E$,

$$
\begin{aligned}
(a * T)(b \cdot u) & =T((b \cdot u) \cdot a)=T(b \cdot(u \cdot a)) \\
& =b \cdot T(u \cdot a)=b \cdot(a * T)(u) .
\end{aligned}
$$

In particular, $\operatorname{Hom}_{A}(A, F)$ is a left $A$-module. If $E=F=A$, then $\operatorname{Hom}_{A}(A, A)=M(A)$ is the usual multiplier algebra of A:

$$
\begin{aligned}
M(A)=\{T \in C L(A, A): T(a b)=a T(b)=T(a) b \\
\forall a, b \in A\},
\end{aligned}
$$

which is a commutative algebra (without $A$ being commutative) and has the identity $I: A \rightarrow A, I(x)=x(x \in A)$.

Lemma 11. Let $\left(A, q_{A}\right)$ a commutative p-normed algebra having a minimal approximate identity, and let $\left(F, q_{F}\right)$ be p-normed space which is an essential isometric A-bimodule. Then, for any $v \in F$,

$$
\left\|L_{v}\right\|_{q_{F}}=\left\|R_{v}\right\|_{q_{F}}=q_{F}(v),
$$

where $L_{v}, R_{v}: A \rightarrow F$ are the maps given by $L_{v}(a)=v \cdot a$ and $R_{v}(a)=a \cdot v, a \in A$.
Proof. Let $v \in F$. Then

$$
\begin{aligned}
\left\|L_{v}\right\|_{q_{A}, q_{F}} & =\sup \left\{q_{F}\left(L_{v}(a)\right): q_{A}(a) \leq 1\right\} \\
& =\sup \left\{q_{F}(v \cdot a): q_{A}(a) \leq 1\right\} \\
& \leq \sup \left\{q_{A}(a) q_{F}(v): q_{A}(a) \leq 1\right\}=q_{F}(v) .
\end{aligned}
$$

On the other hand,

$$
\begin{aligned}
\left\|L_{v}\right\|_{q_{A}, q_{F}} & =\sup \left\{q_{F}(v \cdot a): q_{A}(a) \leq 1\right\} \\
& \geq q_{F}\left(v \cdot e_{\lambda}\right) \quad \forall \lambda \in I,
\end{aligned}
$$

so

$$
\left\|L_{v}\right\|_{q_{A}, q_{F}} \geq \lim _{\lambda} q_{F}\left(v \cdot e_{\lambda}\right)=q_{F}\left(\lim _{\lambda} v \cdot e_{\lambda}\right)=q_{F}(v) .
$$

Hence $\left\|L_{v}\right\|_{q_{A}, q_{F}}=q_{F}(v)$. Similarly, $\left\|R_{v}\right\|_{q_{E}}=q_{E}(v)$.

Lemma 12. Let $\left(A, q_{A}\right)$ a commutative p-normed algebra, and let $\left(F, q_{F}\right)$ be an essential isometric $A$-bimodule. If $A$ has an identity e, then $\operatorname{Hom}_{A}(A, F) \cong F$ and $M(A) \cong A$.

Proof. We claim that

$$
\begin{aligned}
\operatorname{Hom}_{A}(A, F) & \cong\left\{L_{T(e)}: T \in \operatorname{Hom}_{A}(A, F)\right\} \\
& =\left\{L_{v}: v \in F\right\} \cong F .
\end{aligned}
$$

Clearly,

$$
\left\{L_{T(e)}: T \in \operatorname{Hom}_{A}(A, F)\right\} \subseteq\left\{L_{v}: v \in F\right\} \subseteq \operatorname{Hom}_{A}(A, F) .
$$

On the other hand, if $T \in \operatorname{Hom}_{A}(A, F)$, then, for any $a \in A$,

$$
T(a)=T(e a)=T(e) \cdot a=L_{T(e)}(a) .
$$

Hence $T=L_{T(e)}$. Further, by Lemma 11, $\left\|L_{T(e)}\right\|_{q_{A}, q_{F}}=$ $q_{F}(T(e))$. Thus $\operatorname{Hom}_{A}(A, F) \cong F$. In particular, $M(A) \cong$ A.

Density Assumption. In the sequel, we will always assume that, for $X$ a locally compact Hausdorff space and $E$ a topological vector space, $C_{0}(X) \otimes E$ is $u$-dense in $C_{0}(X, E)$. This assumption is crucial for the proof of our main results. For its justification, we mention that as a consequence of the vector-valued versions of Stone-Weierstrass theorem $[8,12$, $15], C_{0}(X) \otimes E$ is $u$-dense in $C_{0}(X, E)$ in each of the following cases.

(a) $E$ is locally convex.

(b) Every compact subset of $X$ has a finite covering dimension and $E$ is any topological vector space.

(c) $E$ is an $F$-space with a basis (e.g., $E=\ell^{p}$ for $p>0$ ).

(d) $E$ has the approximation property. 
Recall that if $T \in M\left(C_{0}(X, A)\right)$, then $T(a \cdot f)=a$. $T(f)$ for $f \in C_{0}(X, A)$ and $a \in A$ ([16, Lemma 4.5]). We also mention that if $\left(A, q_{A}\right)$ is an $p$-normed algebra having a minimal approximate identity, then, by ([16, Lemma 4.4]), $C_{0}(X, A)$ has an approximate identity and hence it is a faithful topological $A$-module. Consequently, for any $T \in$ $M\left(C_{0}(X, A)\right), T(f g)=f T(g)=T(f) g$ for all $f, g \in$ $C_{0}(X, A)$; we will write

$$
\|T\|_{q_{A}}:=\sup \left\{q_{A}(T(f)): f \in C_{0}(X, A),\|f\|_{q_{A}, \infty} \leq 1\right\} .
$$

If $T \in \operatorname{Hom}_{C_{0}(X, A)}\left(C_{0}(X, E), C_{0}(X, F)\right)$, we let

$$
\|T\|_{q_{E}, q_{F}}:=\sup \left\{q_{F}(T(f)): f \in C_{0}(X, E),\|f\|_{q_{E}, \infty} \leq 1\right\} .
$$

Definition 13. Now, let $E=\left(E, q_{E}\right)$ and $F=\left(F, q_{F}\right)$ be $F$ normed spaces. For any closed subspace $U=U_{s}(E, F)$ of $C L(E, F)$ endowed with the strong operator topology $s$, we define

$$
C_{s, b}(X, U)=\{G: X \longrightarrow U:
$$

$G$ is strongly continuous and bounded .

We now define an $F$-norm on $C_{s, b}(X, U)$ by

$$
\|G\|_{C_{s, b}}=\sup _{x \in X}\|G(x)\|_{q_{E}, q_{F}}=\sup _{x \in X} \sup _{u \in E, q_{E}(u) \leq 1} q_{F}(G(x)(u)) .
$$

Then $C_{s, b}(X, U)$ is a complete $p$-normed space under the $p$ norm $\|\cdot\|_{q, \infty}$ defined in (24).

Recall that a left $A$-module $E$ is called faithful (or without order) if, for any $u \in E, a \cdot u=0$ for all $a \in A$ implies that $x=0$ (cf. $[13,14])$.

Lemma 14. Let $A=\left(A, q_{A}\right)$ be a commutative complete $p$ normed algebra, and let $E$ and $F$ be A-modules. Then, for any $T \in \operatorname{Hom}_{C_{0}(X, A)}\left(C_{0}(X, E), C_{0}(X, F)\right)$,

(a) $T(a \cdot f)=a \cdot T(f)$ for $a \in A$ and $f \in C_{0}(X, E)$,

(b) $T(\varphi \cdot f)=\varphi \cdot T(f)$ for $\varphi \in C_{0}(X)$ and $f \in C_{0}(X, E)$.

Proof. (a) We first note that $C_{0}(X)$ is a Banach algebra with a bounded approximate identity, $\left\{\psi_{\alpha}\right\}$ (say). Then, for any $a \in$ $A, u \in E$, and $\varphi \in C_{0}(X)$,

$$
\begin{aligned}
\lim _{\alpha}\left[\left(\psi_{\alpha} \otimes a\right) \cdot(\varphi \otimes u)\right] & =\lim _{\alpha}\left(\psi_{\alpha} \varphi \otimes a \cdot u\right) \\
& =\varphi \otimes a \cdot u=a(\varphi \otimes u) .
\end{aligned}
$$

Since $T \in \operatorname{Hom}_{C_{0}(X, A)}\left(C_{0}(X, E), C_{0}(X, F)\right)$ and $\psi_{\alpha} \otimes a \in$ $C_{0}(X, A), \varphi \otimes u \in C_{0}(X, E)$, we have

$$
\begin{aligned}
T(a \cdot(\varphi \otimes u)) & =\lim _{\alpha} T\left[\left(\psi_{\alpha} \otimes a\right) \cdot(\varphi \otimes u)\right] \\
& =\lim _{\alpha}\left(\psi_{\alpha} \otimes a\right) \cdot T(\varphi \otimes u) \\
& =a \cdot T(\varphi \otimes u) .
\end{aligned}
$$

By $T$ being linear and $C_{0}(X) \otimes E$ being assumed to be $u$-dense in $C_{0}(X, E)$, it follows that $T(a \cdot f)=a \cdot T(f)$ holds for all $f \in C_{0}(X, A)$ and $a \in A$.

(b) Similar to the above part.

We now give the following characterization in the pseudoscaler case by considering both $C_{0}(X)$ and $C_{0}(X, F)$ as $C_{0}(X)$-modules.

Theorem 15. Let $X$ be a locally compact Hausdorff space and $F=\left(F, q_{F}\right)$ a p-normed space. Then

$$
\operatorname{Hom}_{C_{0}(X)}\left(C_{0}(X), C_{0}(X, F)\right) \cong C_{b}(X, F) .
$$

Proof. Let $T \in \operatorname{Hom}_{C_{0}(X)}\left(C_{0}(X), C_{0}(X, F)\right)$ and $x \in X$. If $\varphi, \psi \in C_{0}(X)$ with $\varphi(x) \neq 0$ and $\psi(x) \neq 0$, then there is a neighborhood $N(x)$ of $x$ in $X$ such that

$$
\varphi(t) \neq 0, \quad \psi(t) \neq 0 \quad \text { for any } t \in N(x) .
$$

Since $C_{0}(X)$ is commutative and $C_{0}(X, F)$ is a $C_{0}(X)$-module, following as in $([1, \mathrm{p} .1135])$, we have

$$
\begin{aligned}
\psi(t)(T \varphi)(t) & =T(\psi \cdot \varphi)(t)=T(\varphi \cdot \psi)(t) \\
& =\varphi(t)(T \psi)(t)
\end{aligned}
$$

and then

$$
\frac{T(\psi)(t)}{\psi(t)}=\frac{(T \varphi)(t)}{\varphi(t)} \quad \text { for any } t \in N(x) .
$$

Now, for each $x \in X$ with $\varphi(x) \neq 0$, define $g_{T}: X \rightarrow F$ by

$$
g_{T}(x)=\frac{(T \varphi)(x)}{\varphi(x)} .
$$

By the above argument, the function $g_{T}(x)$ defined in this way is independent of the choice of $\varphi \in C_{0}(X)$; hence $g_{T}$ is welldefined.

Clearly if $\varphi(x) \neq 0$, then $(T \varphi)(x)=g_{T}(x) \varphi(x)$. The equality also holds when $\varphi(x)=0$. [To see this, choose $\psi \in C_{0}(X)$ such that $\psi(x) \neq 0$. Then

$$
\psi(x)(T \varphi)(x)=T(\psi \varphi)(x)=\varphi(x)(T \psi)(x)=0,
$$

and so $T \varphi(x)=0$.]

Next, $g_{T} \in C_{b}(X, F)$, as follows. For any $x \in X$ with $\varphi(x) \neq 0$, by Urysohn's lemma, we can choose a $\varphi \in C_{0}(X)$ such that $\|\varphi\|_{\infty}=|\varphi(x)|$. So

$$
q_{F}\left[g_{T}(x)\right]=\frac{q_{F}[T \varphi(x)]}{|\varphi(x)|} \leq \frac{\|T\|_{q_{F}}\|\varphi\|_{\infty}}{|\varphi(x)|}=\|T\|_{q_{F}}
$$

for all $x \in X$. Hence $\left\|g_{T}\right\|_{q, \infty} \leq\|T\|_{q_{F}}$, and so $g_{T} \in C_{b}(X, F)$. On the other hand, since

$$
q_{F}[(T \varphi)(x)]=q_{F}\left[g_{T}(x) \varphi(x)\right] \leq\left\|g_{T}\right\|_{q, \infty}\|\varphi\|_{\infty},
$$

we have $\|T\|_{q_{F}} \leq\left\|g_{T}\right\|_{q, \infty}$. Consequently $\left\|g_{T}\right\|_{q_{F}, \infty}=\|T\|_{q_{F}}$. This shows that $\operatorname{Hom}_{C_{0}(X)}\left(C_{0}(X), C_{0}(X, F)\right)$ is isometrically embedded in $C_{b}(X, F)$. 
Conversely, for any $g \in C_{b}(X, F)$, we define $T_{g}$ : $C_{0}(X) \rightarrow C_{0}(X, F)$ by

$$
T_{g}(\varphi)=g \cdot \varphi, \varphi \in C_{0}(X) .
$$

Then one can easily show that $T_{q}$ is a multiplier from $C_{0}(X)$ to $C_{0}(X, F)$ and that $\|g\|_{q, \infty}=\left\|T_{g}\right\|_{q_{F}}$.

Now we can establish the main theorem by considering both $C_{0}(X, E)$ and $C_{0}(X, F)$ as $C_{0}(X, A)$-modules.

Theorem 16. Let $A=\left(A, q_{A}\right)$ be a commutative complete $p$ normed algebra, and let $E=\left(E, q_{E}\right)$ and $F=\left(F, q_{F}\right)$ be $p$ normed spaces which are also essential isometric A-modules. Then

$$
\operatorname{Hom}_{C_{0}(X, A)}\left(C_{0}(X, E), C_{0}(X, F)\right) \cong C_{s, b}\left(X, \operatorname{Hom}_{A}(E, F)\right) .
$$

The correspondence between the multiplier $T$ and the function $G$ is given by the following relation:

$$
\begin{array}{r}
(T f)(x)=G(x) \cdot f(x) \\
\text { for } x \in X \text { and any } f \in C_{0}(X, E) .
\end{array}
$$

Proof. Let $T \in \operatorname{Hom}_{C_{0}(X, A)}\left(C_{0}(X, E), C_{0}(X, F)\right)$. Then we can

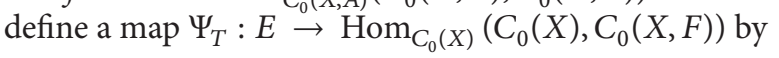

$$
\Psi_{T}(u)(\varphi)=T(\varphi \otimes u) \quad \text { for } u \in E, \varphi \in C_{0}(X) .
$$

To see that this map is well-defined, first note that $\Psi_{T}(u)(\varphi) \epsilon$ $C_{0}(X, F)$. For a fixed $u \in E$, the operator $\Phi_{T}(u)$ defines a bounded linear operator from $C_{0}(X)$ into $C_{0}(X, F)$, since by (46),

$$
\begin{aligned}
\left\|\Psi_{T}(u)(\varphi)\right\|_{q_{E}, \infty} & =\|T(\varphi \otimes u)\|_{q_{E}, \infty} \\
& \leq\|T\|_{q_{E}} \cdot\|\varphi \otimes a\|_{q_{E}, \infty}
\end{aligned}
$$

further, it is a multiplier since, for any $\varphi, \psi \in C_{0}(X)$,

$$
\Psi_{T}(u)(\varphi \psi)=T(\varphi \psi \otimes u)=\varphi \cdot T(\psi \otimes u) .
$$

Hence $\Psi_{T}(u) \in \operatorname{Hom}_{C_{0}(X)}\left(C_{0}(X), C_{0}(X, F)\right)$. By Theorem 15 , there exists an element, say $g_{u}$, in $C_{b}(X, F)$ such that

$$
\Psi_{T}(u)(\varphi)=g_{u} \cdot \varphi, \quad \text { for } u \in E, \varphi \in C_{0}(X) .
$$

Now, we can define a map $G: X \rightarrow \operatorname{Hom}_{A}(E, F)$ by

$$
G(x)(u)=g_{u}(x) \quad \text { for } x \in X, u \in E .
$$

To see that this map is well-defined, first note that, for a fixed $x \in X, G(x)$ is a linear operator from $E$ into $F$. Moreover, for $a \in A$ and $\varphi \in C_{0}(X)$, we have

$$
\begin{aligned}
G(x)(a \cdot u) \cdot \varphi(u) & =g_{a u}(x) \varphi(x)=T(\varphi \otimes a \cdot u)(x) \\
& =a \cdot T(\varphi \otimes u)(x)=a \cdot g_{u}(x) \varphi(x) \\
& =a \cdot G(x)(u) \varphi(x),
\end{aligned}
$$

or

$$
G(x)(a \cdot u)=a \cdot G(x)(u) .
$$

This implies that $G(x) \in \operatorname{Hom}_{A}(E, F)$, and hence $G \in$ $C_{s, b}\left(X, \operatorname{Hom}_{A}(E, F)\right)$. Next we establish isometry between $T$ and $G$. For $x \in X$ and $\varphi \otimes u \in C_{0}(X) \otimes E$ with $\|\varphi \otimes u\|_{q_{E}, \infty} \leq 1$,

$$
\begin{aligned}
\|G(x)\|_{q_{E}, q_{F}} & =\sup _{q_{E}(u) \leq 1} q_{F}[G(x)(u)]=\sup _{q_{E}(u) \leq 1} q_{F}\left[g_{u}(x)\right] \\
& \leq \sup _{q_{E}(u) \leq 1}\left\|g_{u}\right\|_{q_{F}, \infty}=\sup _{\substack{q_{E}(u) \leq 1 \\
\|\varphi\|_{\infty} \leq 1}}\left\|g_{u} \cdot \varphi\right\|_{q_{F}, \infty} \\
& =\sup _{\|\varphi \otimes u\|_{q_{E}, \infty} \leq 1}\|T(\varphi \otimes u)\|_{q_{F}, \infty}=\|T\|_{q_{E}, q_{F}},
\end{aligned}
$$

since $C_{0}(X) \otimes E$ is $u$-dense in $C_{0}(X, E)$. So $\|G\|_{C_{s, b}} \leq\|T\|_{q_{E}, q_{F}}$. But

$$
\begin{aligned}
\|T(\varphi \otimes u)\|_{q_{F}, \infty} & =\left\|g_{u} \cdot \varphi\right\|_{q_{F}, \infty} \leq\left\|g_{u}\right\|_{q_{F}, \infty}\|\varphi\|_{\infty} \\
& \leq\|G\|_{C_{s, b}}\|u\|\|\varphi\|_{\infty}=\|G\|_{C_{s, b}}\|\varphi \otimes u\|_{q_{E}, \infty}
\end{aligned}
$$

for all $\varphi \otimes u \in C_{0}(X) \otimes E$. Consequently, $\|T\|_{q_{E}, q_{F}} \leq\|G\|_{C_{s, b}}$.

Conversely, let $G \in C_{s, b}\left(X, \operatorname{Hom}_{A}(E, F)\right)$ and $\varphi \in C_{0}(X)$. Then $G \cdot \varphi$ is a continuous function on $X$ given by

$$
(G \cdot \varphi)(x)(u)=(G(x) u) \varphi(x), \quad x \in X, u \in E .
$$

It is easy to see that $G \cdot \varphi$ vanishes at infinity, and so $G \cdot \varphi \in$ $C_{0}\left(X, \operatorname{Hom}_{A}(E, F)\right)$. For any $u \in E$ and $\varphi \in C_{0}(X), G$ determines a bounded linear operator $T$ from $C_{0}(X, E)$ to $C_{0}(X, F)$ given by

$$
T(\varphi \otimes u)(x)=(G(x) u) \varphi(x) .
$$

Again, since $C_{0}(X) \otimes E$ is $u$-dense in $C_{0}(X, E)$, it follows that $\|T\|_{q_{E}, q_{F}}=\|G\|_{C_{s, b}}$.

Since $E$ and $F$ are $A$-modules, for any $h \otimes a \in C_{0}(X) \otimes A$ and $\varphi \otimes u \in C_{0}(X) \otimes E$,

$$
\begin{aligned}
T((h \otimes a) \cdot(\varphi \otimes u)) & =T(h \varphi \otimes a u) \\
& =G(\cdot)(a \cdot u)(h \varphi)(\cdot) \\
& =a \cdot h(\cdot) G(\cdot)(u) \varphi(\cdot) \\
& =(h \otimes a) \cdot T(\varphi \otimes u) .
\end{aligned}
$$

Hence $T$ is a multiplier on $C_{0}(X, E)$ since $C_{0}(X) \otimes E$ is $u$-dense in $C_{0}(X, E)$. The isometry between $G$ and $T$ now implies that

$$
\operatorname{Hom}_{C_{0}(X, A)}\left(C_{0}(X, E), C_{0}(X, F)\right) \cong C_{s, b}\left(X, \operatorname{Hom}_{A}(E, F)\right) .
$$

\section{Applications}

As an application of the above results, in particular of Theorem 16, we can deduce several known results, as follows. 
Corollary 17 (see [3]). Let $X$ be a locally compact Hausdorff space and $A=(A,\|\cdot\|)$ a commutative Banach algebra, and let $E$ and $F$ be Banach $A$-modules. Then

$$
\operatorname{Hom}_{C_{0}(X, A)}\left(C_{0}(X, E), C_{0}(X, F)\right) \cong C_{s, b}\left(X, \operatorname{Hom}_{A}(E, F)\right) .
$$

Corollary 18 (see $[3,5])$. Let $X$ be a locally compact Hausdorff space and $A=(A,\|\cdot\|)$ be a commutative Banach algebra with identity of norm 1 , and let $E$ be a Banach A-module. Then

$$
\operatorname{Hom}_{C_{0}(X, A)}\left(C_{0}(X, E), C_{0}(X, E)\right) \cong C_{b}(X, E) .
$$

Corollary 19 (see [16]). Let $X$ be a locally compact Hausdorff space and $A=(A, q)$ a commutative complete p-normed algebra with a minimal approximate identity. Then

$$
M\left(C_{0}(X, A)\right) \cong C_{s, b}\left(X, M(A)_{u}\right) .
$$

Proof. This follows from the fact that $\operatorname{Hom}_{A}(A, A)=M(A)$.

Corollary 20 (see [1]). Let X be a locally compact Hausdorff space. Then

$$
M\left(C_{0}(X)\right) \cong C_{b}(X)
$$

Proof. This follows from the fact that $\operatorname{Hom}_{C_{0}(X)}\left(C_{0}(X)\right.$, $\left.C_{0}(X)\right) \cong C_{b}(X)$.

Example 21. Let $A_{p}, 0<p \leq 1$, denote the algebra of all holomorphic functions in the unit $\operatorname{disc} D=\{z \in \mathbb{C}:|z| \leq 1\}$ :

$$
\varphi(z)=\sum_{n=0}^{\infty} a_{n} z^{n}, \quad z \in D
$$

for which

$$
\|\varphi\|_{p}=\sum_{n=0}^{\infty}\left|a_{n}\right|^{p}<\infty .
$$

This is a commutative complete $p$-normed algebra with the pointwise multiplication and has an identity ([7, p. 135]; [17, p. 8]). In this case,

$$
M\left(C_{0}\left(X, A_{p}\right)\right) \simeq C_{b}\left(X, M\left(A_{p}\right)_{s}\right) \simeq C_{b}\left(X, A_{p}\right) .
$$

\section{Conflict of Interests}

The authors declare that there is no conflict of interests regarding the publication of this paper.

\section{Acknowledgments}

This work was funded by the Deanship of Scientific Research (DSR), King Abdulaziz University, Jeddah, under the Project no. 3-059/429. The authors therefore acknowledge with thanks DSR technical and financial support. The authors are also grateful to the referee for his many useful suggestions to improve the paper.

\section{References}

[1] J.-K. Wang, "Multipliers of commutative Banach algebras," Pacific Journal of Mathematics, vol. 11, pp. 1131-1149, 1961.

[2] C. A. Akemann, G. K. Pedersen, and J. Tomiyama, "Multipliers of $C^{*}$-algebras," Journal of Functional Analysis, vol. 13, pp. 277301,1973

[3] J. C. Candeal Haro and H. C. Lai, "Multipliers in continuous vector-valued function spaces," Bulletin of the Australian Mathematical Society, vol. 46, no. 2, pp. 199-204, 1992.

[4] H. C. Lai, "Multipliers of a Banach algebra in the second conjugate algebra as an idealizer," The Tohoku Mathematical Journal, vol. 26, pp. 431-452, 1974.

[5] H. C. Lai, "Multipliers for some spaces of Banach algebra valued functions," The Rocky Mountain Journal of Mathematics, vol. 15, no. 1, pp. 157-166, 1985.

[6] H. C. Lai, "Multipliers of Banach valued function spaces," Australian Mathematical Society A, vol. 39, no. 1, pp. 51-62, 1985.

[7] A. Mallios, Topological Algebras-Selected Topics, vol. 124 of North-Holland Mathematics Studies, North-Holland, New York, NY, USA, 1986.

[8] A. H. Shuchat, "Approximation of vector-valued continuous functions," Proceedings of the American Mathematical Society, vol. 31, pp. 97-103, 1972.

[9] G. Köthe, Topological Vector Spaces I, Springer, New York, NY, USA, 1969.

[10] S. Rolewicz, Metric Linear Spaces, D. Reidel, Dordrecht, The Netherlands, 1985.

[11] M. Adib, A. H. Riazi, and L. A. Khan, "Quasimultipliers on Falgebras," Abstract and Applied Analysis, vol. 2011, Article ID 235273, 30 pages, 2011.

[12] L. A. Khan, Linear Topological Spaces of Continuous VectorValued Functions, Academic Publications, 2013.

[13] M. A. Rieffel, "Induced Banach representations of Banach algebras and locally compact groups," Journal of Functional Analysis, vol. 1, pp. 443-491, 1967.

[14] L. A. Khan, "Topological modules of continuous homomorphisms," Journal of Mathematical Analysis and Applications, vol. 343, no. 1, pp. 141-150, 2008.

[15] M. Abel, "Topological bimodule-algebras," in Proceedings of the 3rd International Conference on Topological Algebra and Applications, pp. 25-42, Oulu, Finland, 2004.

[16] L. A. Khan and S. M. Alsulami, "Multipliers of commutative Falgebras ofcontinuous vector-valued functions," Bulletin of the Malaysian Mathematical Sciences Society. In press.

[17] W. Żelazko, "Metric generalizations of Banach algebras," Dissertations Mathematics, vol. 47, 70 pages, 1965. 


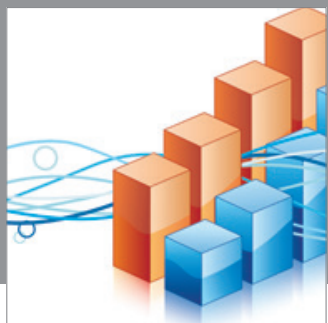

Advances in

Operations Research

mansans

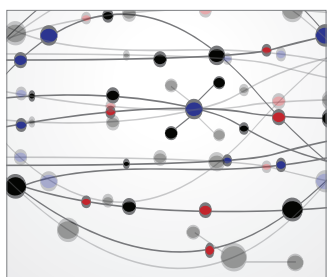

The Scientific World Journal
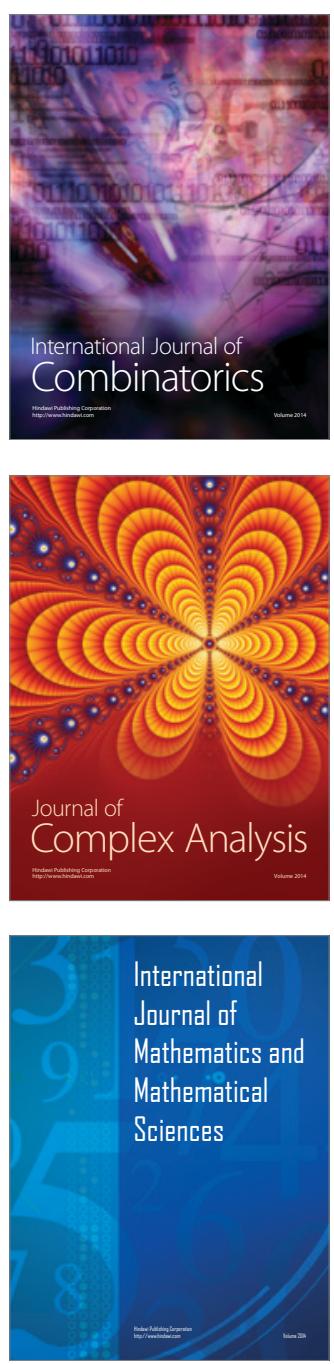
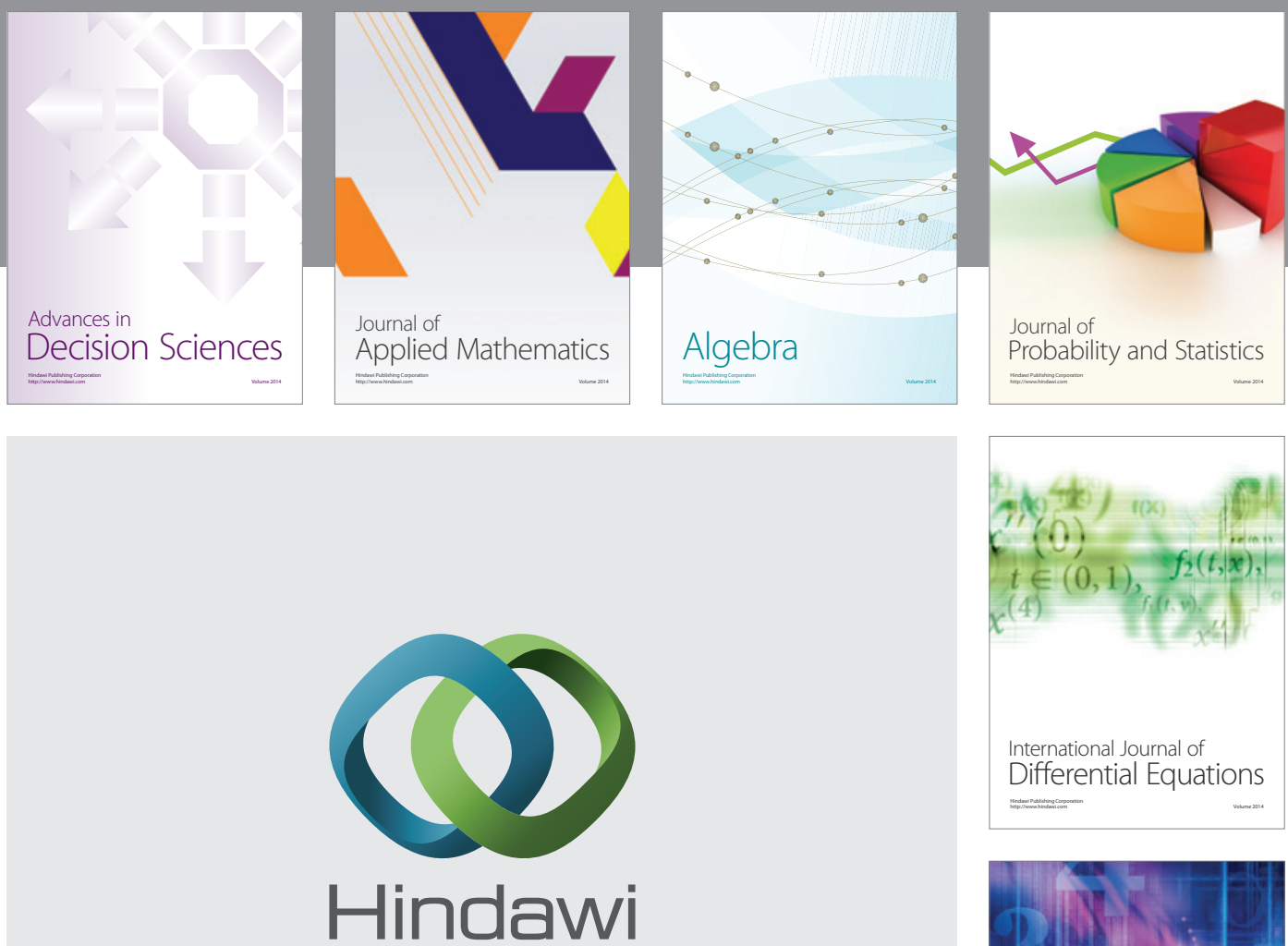

Submit your manuscripts at http://www.hindawi.com
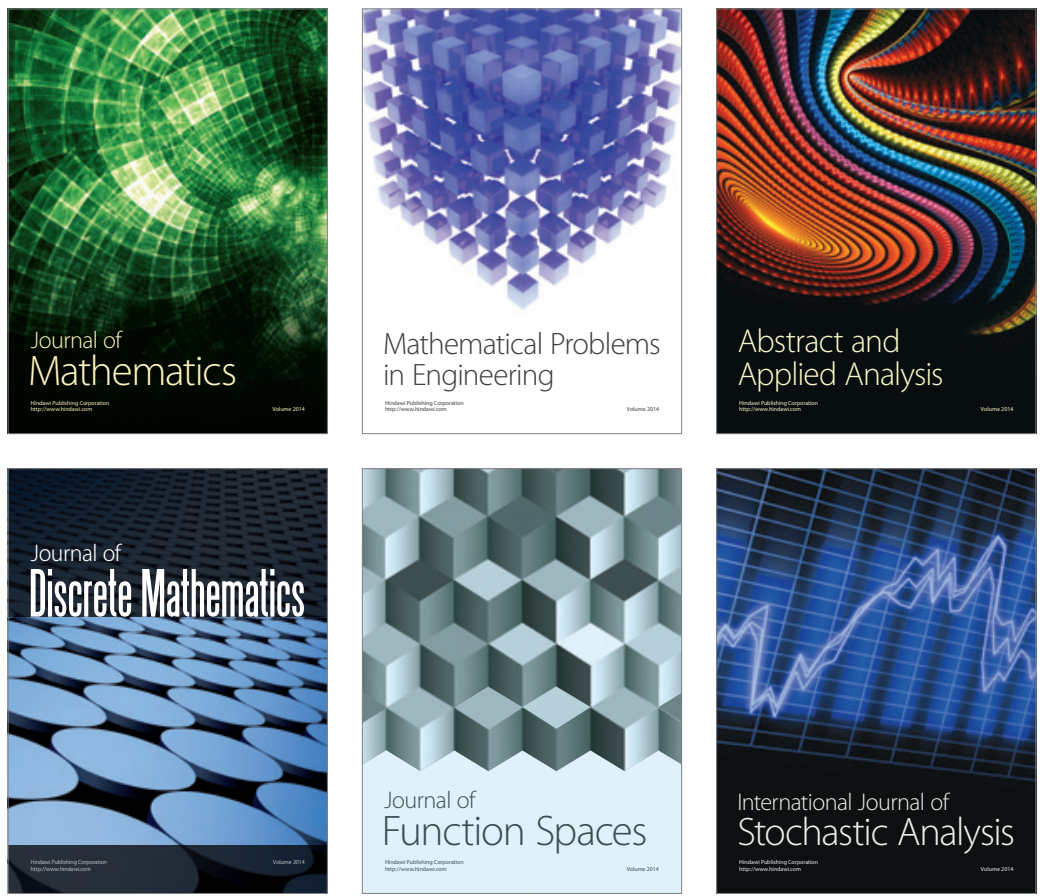

Journal of

Function Spaces

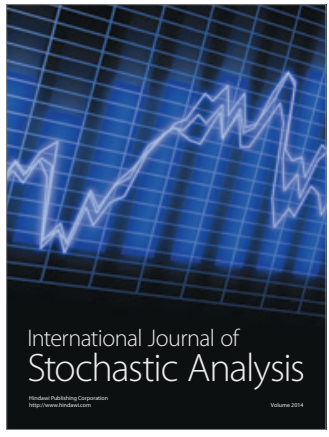

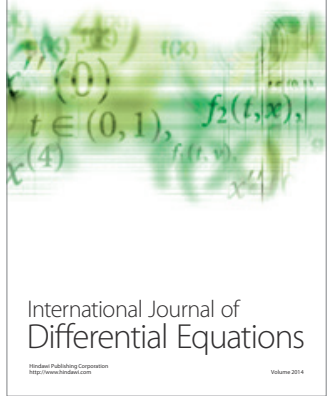
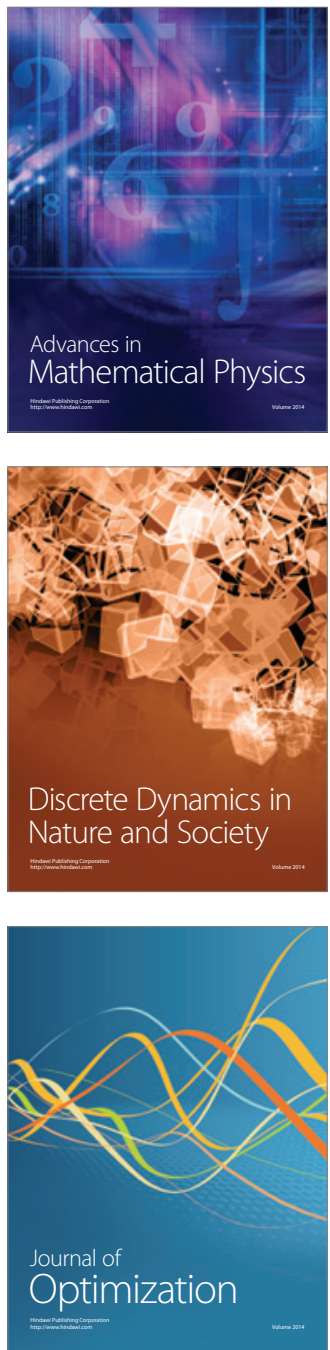